\title{
A Preclinical Evaluation Comparing the Performance of a Novel 19-G Flexible Needle to a Commercially Available 22-G EBUS-TBNA Sampling Needle
}

\author{
Kasia Czarnecka-Kujawa ${ }^{a}$ b Alain Tremblay ${ }^{c}$ Kazuhiro Yasufuku ${ }^{b}$ \\ Anna Sczaniecka ${ }^{d}$ Sujeeth Parthiban ${ }^{d}$ Lawrence Kunz $^{d}$ David Dillard ${ }^{d}$ \\ Xavier Gonzalez ${ }^{d}$ \\ Divisions of a Respirology and ${ }^{\mathrm{b}}$ Thoracic Surgery, Toronto General Hospital, University Health Network, University \\ of Toronto, Toronto, ON, and ' ${ }^{\mathrm{D}}$ ivision of Respirology, Foothills Hospital, University of Calgary, Calgary, AB, Canada; \\ 'Spiration, Redmond, WA, USA
}

\section{Keywords}

Endobronchial ultrasound-guided transbronchial needle aspiration needle · Granulomatous inflammation .

Interventional pulmonology

\begin{abstract}
Background: Needle samples may not provide sufficient diagnostic material for the assessment of mediastinal lymph nodes. Objective: The study compared the specimen size and diagnostic performance of a new 19-G endobronchial ultrasound-guided transbronchial needle aspiration (EBUSTBNA) needle to that of a standard 22-G EBUS-TBNA needle in a swine model of granulomatous lymphadenopathy. Methods: Granulomatous inflammation was induced in mediastinal lymph nodes (LNs) of 10 domestic swine by injection of talc slurry. The affected LNs were sampled with the 19- and 22-G needles. Collected core tissue area and volume were determined using a specialized software and known needle internal diameter. The sample's quality was assessed
\end{abstract}

using the biopsy core morphology grade (BCMG) as well as the biopsy diagnostic correlation grade (BDCG). Results: There was a significant increase in the average LN size from baseline $(11.6 \pm 3.2$ to $15.2 \pm 3.8 \mathrm{~mm} ; p<0.001)$ after talc injection. A total of 132 paired samples were collected from 38 LNs. The average mass and volume of the 19-G needle sample were larger than those of the 22-G needle sample: 33.78 \pm 47.48 vs. $25.18 \pm 32.08 \mathrm{mg}(p<0.002)$ and $11.40 \pm 13.91$ vs. $6.91 \pm 6.42 \mathrm{~mm}^{3}(p<0.0004)$, respectively. The pooled needle biopsy samples for the 19- and the 22-G needles had similar BCMG (1.38 \pm 0.86 vs. $1.43 \pm 0.87, p>0.2)$ and BDCG (1.54 \pm 0.93 vs. $1.57 \pm 0.93, p>0.2$ ). The $19-G$ needle samples tended towards less blood contamination ( $p=0.057)$, more often granuloma identification ( 46 vs. $32 \%, p=0.2$ ) and had more cartilage contamination $(0.49 \pm 1.46$ vs. $4.81 \pm 16.49 \% p<$ $0.003)$. Conclusion: In experienced hands, the 19- and the 22-G EBUS-TBNA needles have a similar diagnostic yield in the swine model of granulomatous lymphadenopathy. The samples collected by the 19-G needle are larger and may have less blood contamination.

\section{KARGER}

(c) 2017 S. Karger AG, Basel

E-Mail karger@karger.com

www.karger.com/res
Kasia Czarnecka-Kujawa, MD, FRCPC

Division of Respirology, Division of Thoracic Surgery Toronto General Hospital, University Health Network 585 University Ave., 9N-941, Toronto, ON M5G 2N2 (Canada)

E-Mail kasia.czarnecka@uhn.ca 


\section{Introduction}

In the era of personalized medicine, determining the molecular signature of lung cancer has become the standard of care $[1,2]$. Endobronchial ultrasound-guided transbronchial needle aspiration (EBUS-TBNA) is the recommended first test in mediastinal lymph node (LN) staging in patients with primary lung cancer [3]. In patients with proximal tumors in contact with major airways, EBUS-TBNA can offer diagnosis of the primary tumor and it often is the only invasive diagnostic test performed that guides therapeutic decisions in many patients with advanced disease $[4,5]$.

EBUS-TBNA samples obtained with 22- or 21-G needles show no significant difference in diagnostic accuracy. However, the 21-G needle provides a better detail of the histological structure which can be useful in the assessment of lymphoma [6]. EBUS-TBNA samples provide sufficient quantity and quality of material for molecular testing. Reported adequacy of EBUS-TBNA samples for molecular diagnosis ranges from 77 to $98 \%[7,8]$. However, as more cancer-related genes continue to be identified as potential therapy targets in lung cancer, more tissue may be required for molecular analysis. Current EBUS-TBNA is limited in the amount of tissue that it can provide for such analyses. Sometimes the procedure needs to be repeated or the patient may need to be subjected to mediastinoscopy to provide larger samples of LN tissue to guide therapy. The diagnostic yield of EBUSTBNA has also been shown to be lower in lymphoma and fibrotic disease such as sarcoidosis [9-12]. The 19-G ProCore needle used with endoscopic ultrasound-guided fine needle aspiration (EUS-FNA) to assess gastrointestinal tumors has been shown to obtain diagnostic samples with fewer needle passes than the 22-G needle [13]. A larger needle bore may also provide more tissue during EBUS-TBNA to allow for better histologic and molecular analysis than the currently used 22-G EBUS-TBNA needle. In addition, diagnosis in neoplasms such as stromal tumors and lymphoma requires diagnostic samples to provide information on tumor morphology and architecture to ensure accurate pathologic assessment.

Recently, a new EBUS needle has become available, the 19-G ViziShot FLEX needle (Olympus Respiratory America, Redmond, WA, USA), which has a larger inner lumen and the potential to collect larger tissue samples while simultaneously preserving the histological integrity of collected material. Preliminary assessment of this needle's performance in a small sample of patients with mediastinal lymphadenopathy showed good diagnostic yield in a

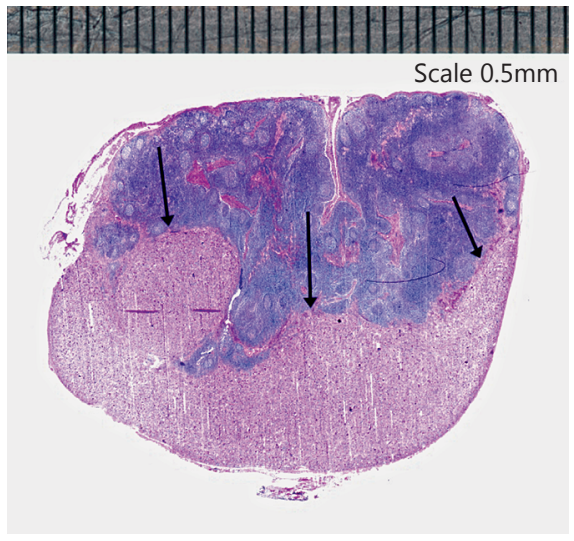

Fig. 1. Hematoxylin and eosin stain of talc-induced nodular histiocytic granulomatous lymphadenopathy, in subcapsular location (below the arrows), which was circumscribed, with minimal to mild encapsulation, and phagocytized infusate talc crystals. Remaining lymph node area (upper location) had mild sinusoidal histiocytosis without infusate crystals present consistent with normal lymph node architecture. Magnification $\times 1$.

variety of different pathologies but without comparison to the standard 22-G EBUS-TBNA needle [14].

The goal of this study was to determine the utility of this novel 19-G EBUS-TBNA needle by comparing it to the more commonly used $22-\mathrm{G}$ needle with respect to specimen size and diagnostic yield by using an animal model of granulomatous lymphadenopathy.

\section{Methods}

The study protocol was developed in collaboration between preclinical scientists and clinical interventional bronchoscopists. The protocol was reviewed and approved by the Testing Site's Institutional Animal Care and Use Committee (IACUC) for compliance with regulatory guidelines concerning the care and use of animals (IACUC protocol \# $140715 \mathrm{MP}$ ).

\section{Model Preparation}

Ten healthy domestic swine $(39.3 \pm 1.7 \mathrm{~kg})$ were used. The bronchoscopic EBUS procedures were performed under general anesthesia. After the paratracheal and mediastinal LN stations were located and measured using ultrasound, 3-4 of the LNs were selected and injected with a small amount of talc slurry using a ViziShot 22-G needle to induce granuloma formation (Fig. 1). All animals were recovered and given a minimum of 6 weeks for the lymphadenopathy to mature.

\section{In vivo Evaluations and Sampling}

After the maturation period, animals were returned to the procedural room, intubated, and placed under general anesthesia. After general airway inspection, talc-affected LNs and anatomical landmarks were identified. EBUS was used to systematically mea- 
sure the LNs to quantify changes produced by the inflammatory process. EBUS-TBNA sampling was performed by 3 clinicians experienced in EBUS-TBNA (K.Y., K.C.-K., and A.T.). A standardized sample collection technique was used by the clinicians. For each animal, 2 new 22-G and 2 new 19-G EBUS-TBNA needles were used for the sample collection. Clinicians consistently applied $20 \mathrm{~mL}$ of vacuum with a locking syringe and performed 20 agitations when collecting a sample within a given LN. Samples were ejected using both air and the stylet. The needle sequence for sampling was selected using a true random sequence generator (www.random.org) for the first LN, and alternated in each subsequent LN.

All samples were processed immediately and according to a standardized protocol. First, each sample was ejected into a chamber slide containing a small amount of ThinPrep ${ }^{\circledR}$ CytoLyt ${ }^{\circledR}$ Solution (Hologic, Bedford, MA, USA). Sample mass was immediately determined using a precision scale. Then, each sample was photographed while still in the chamber slide. The cores were separated and placed in formalin and the remaining cellular material was placed in a centrifuge tube with CytoLyt ${ }^{\circledR}$. Samples collected by each needle from individual LNs were pooled in the corresponding tubes after imaging. The material in CytoLyt was spun down into a pellet. All materials were transferred to the study cytopathologist who remained blinded to the device used to collect each sample. No immediate complications occurred. After the EBUS-TBNA procedures, the animals recovered and were observed for a minimum of 2 weeks.

The photographic images of samples were used to quantify core tissue area using image analysis software (ImageJ, NIH, Bethesda, MD, USA). The volume was calculated from the area, using the inner diameter of the respective needles.

\section{Final Follow-Up, Necropsy, and Tissue Processing}

Prior to necropsy, the animal airways were examined with conventional bronchoscopy to look for delayed airway complications. Animals were sacrificed at the end of the study and complete thoracic necropsies were performed. The lungs were infused with formalin via tracheal pressure perfusion and the entire lungs, including the LNs, were collected. Specimens were placed in formalin and provided to the pathologist to be sectioned and processed into histological slides.

\section{Histology and Cytology Evaluations}

The pooled formalin-fixed needle core samples (biopsies) were processed into hematoxylin-and-eosin-stained slides. The pellet samples were processed to create smears with Giemsa stains for cytology. The study pathologist performed sectioning through the LN and surrounding tissues, and these slices were processed into hematoxylin-and-eosin-stained slides for histopathology. The needle samples were evaluated histopathologically and compared to the LNs from which they were taken. Each pair of samples (samples collected from each LN with the 19- and the 22-G needles) was evaluated for the difference in sample morphology and diagnostic grade.

Each of the tissue samples was assigned a biopsy core morphology grade (BCMG) which was adapted from the study by Shackelford et al. [15] with modification to fit our model by the blinded study pathologist (Table 1). This grade is based entirely on the morphologic appearance of the collected biopsy tissues and not the types of cells contained within the samples.

19-G versus 22-G EBUS-TBNA Needles:

Diagnostic Comparison
Table 1. Biopsy sample morphological correlation grade scale

1 Good collection: needle collects suitable size samples that allow histological characterization of the site from which the sample was collected, and is free of cellular morphological distortion and/or disruption

2 Fair collection: needle collects a sample that is fragmented into small core pieces and single to aggregated clusters of cells and tissue stroma, and has less than 5\% of the tissue/ cells morphologically distorted or disrupted but characterization of the sample collection site is possible

3 Poor collection: needle collects a sample that is fragmented into small core pieces and single to aggregated clusters of cells and tissue stroma, and has less than $20 \%$ of the tissue/ cells morphologically distorted or disrupted but characterization of the sample collection site is possible

4 Unacceptable collection: needle does not collect enough tissue samples, or enough free of morphological distortion and/or disruption to be useful in characterization or analysis of the biopsy, either histologically or cytologically

Table 2. Biopsy sample diagnostic correlation grade scale

1 Good correlation: all morphological diagnostic features of the biopsy target tissue were detected with the biopsy sample

2 Fair correlation: the biopsy lacked morphological features, or had damaged cellular features, which resulted in an incomplete diagnosis or one that characterized most of the site tissue, but was not definitive

3 Poor correlation: the needle biopsy collected a sample containing distorted, disrupted, fragmented, or small core pieces to single or aggregated clusters of cells and tissue stroma, which lacked the key cell types or morphology required to make a complete diagnosis. This type of biopsy results in a description of cellular elements (cytology) with a list of possible diagnosis

4 Unacceptable correlation: the needle biopsy did not sample the target site, did not collect enough tissue sample, or enough free of morphological distortion and/or disruption to be useful in characterization or analysis of the biopsy, either histologically or cytologically

The biopsy samples were also assigned a biopsy diagnostic correlation grade (BDCG) (Table 2). The BDCG developed for this study was adapted from 1 observation in the study by Chee et al. [11] with modifications to fit our granulomatous inflammation model. The BDCG reflects how representative the sample tissue is of the cellular morphology of the LN target tissue site (clinically the presence of target tissue in the collected sample would be necessary to make the diagnosis). 


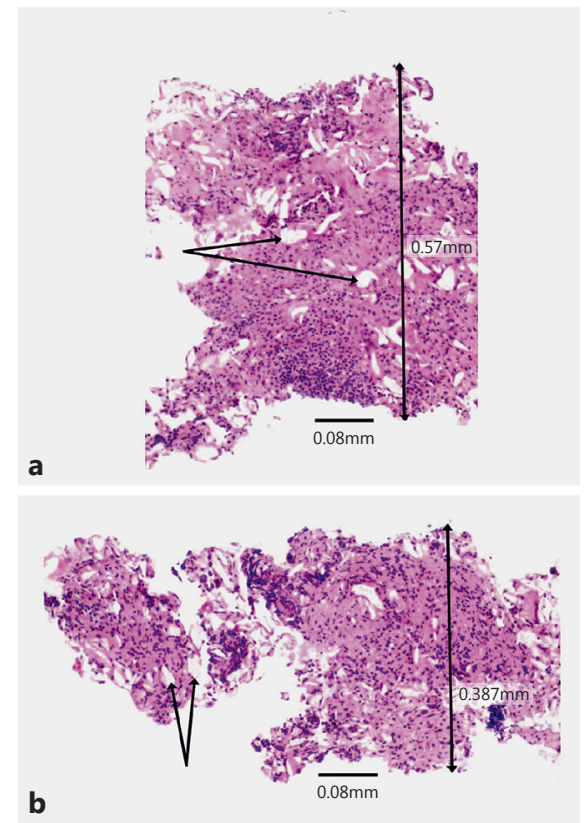

Fig. 2. Hematoxylin and eosin stain of the biopsy cores from the $19-\mathrm{G}$ (a) and 22-G (b) needles, with the 19-G being larger in diameter and in general has more discernible tissue architecture and providing more stroma that decreases the core fragmentation compared to the 22-G needle. The two branched arrows in both core images point to phagocytized infusate talc crystals within multinucleated macrophages. Magnification $\times 100$.

\section{Statistical Methods}

Means were used to describe continuous variables. For withinsubject (i.e., between-needle) comparisons of sample mass, volume, BCMG, and BDCG, a nonparametric Wilcoxon signed-rank test was used. Hypothesis testing was 2-sided, using an alpha level of 0.05. Analyses were conducted using Microsoft Excel (Microsoft Office 365 ProPlus Version 15.0.4911.1002). In addition, the $\mathrm{McNemar}$ test was used to compare the binary outcomes (i.e., presence or absence of granuloma in the collected samples).

The study was performed between January and May of 2016 at the BIODEVELOPMENT Inc. in Stanwood, WA, USA.

\section{Results}

A total of 38 LNs were sampled in 10 animals. Each of the clinicians performed sampling in at least 5 animals, typically in stations R2, R4, L4, and L7. A total of 132 paired samples were collected. There were no immediate procedure-related complications.

There was a statistically significant increase in the average LN size in the talc-affected LNs from a baseline of $11.6 \pm 3.2$ to $15.2 \pm 3.8 \mathrm{~mm}(p<0.001)$. The analysis of the paired EBUS-TBNA samples showed that the average mass of the samples collected by the 19-G needle was greater than that of the samples collected by the $22-\mathrm{G}$ needle (33.78 \pm 47.48 vs. $25.18 \pm 32.08 \mathrm{mg}$, respectively, $p<$ 0.002) (Fig. 3).

The calculated core tissue volume collected by the $19-G$ needle was higher than that of the samples collected by the $22-\mathrm{G}$ needle $\left(11.40 \pm 13.91\right.$ vs. $6.91 \pm 6.42 \mathrm{~mm}^{3}$, $p<0.0004$ ) (Fig. 2). The pooled needle biopsy samples for the $19-\mathrm{G}$ needles and the $22-\mathrm{G}$ needles had similar BCMG $(1.38 \pm 0.86$ vs. $22 \mathrm{G} 1.43 \pm 0.87, p>0.2)$ and the BDCG were also not significantly different $(1.54 \pm 0.93$ vs. $22 \mathrm{G}$ $1.57 \pm 0.93, p>0.2)$. Both, the BCMG and BDCG were determined by the pathologist as having a "good correlation" with the final LN pathology for both needle types.

The pooled cell/debris pellets and the smears from the needle samples for the 19- and the 22-G needle had similar morphologic grades $(3.19 \pm 0.95$ vs. $2.83 \pm 1.18, p>0.1$ for debris pellet; $3.45 \pm 0.83$ vs. $3.32 \pm 0.84, p>0.2$ for the debris smears) and diagnostic grades (3.28 \pm 0.97 vs. 3.03 $\pm 1.08, p>0.2$ for the debris pellet; $3.63 \pm 0.75$ vs. $3.42 \pm$ 0.83 for the debris smears) were both rated as having a "poor correlation" with the final pathology sample.

There was a tendency for the $22-G$ needle sample to contain more blood $(p=0.057)$. Samples collected by the $22-G$ needle contained less cartilage than the samples collected by the $19-\mathrm{G}$ needle $(0.49 \pm 1.46$ vs. $4.81 \pm 16.49 \%$, $p<0.003$ ) (Table 3). Granulomas were identified more frequently in the samples collected by the $19-G$ needle, but this difference did not reach statistical significance (46 vs. $32 \%$ of specimens, $p=0.2$ ).

\section{Discussion}

Our study is the first trial documenting diagnostic performance of the ViziShot 19-G FLEX EBUS and ViziShot 22-G EBUS needles, in an animal model of granulomatous lymphadenopathy. The animal model was adequate, relevant and allowed for direct comparisons and standardization of methods which may be difficult to achieve in clinical studies.

Our study did not demonstrate a difference in either the histological or cytological yield of the samples collected by the 2 needles. However, binary analysis of the data for the presence or absence of granuloma showed that the $19-\mathrm{G}$ needle more frequently had positive results, although this difference was not statistically significant.

Because the sampling technique was standardized, it is likely that the larger mass and sample volumes obtained by the $19-\mathrm{G}$ needle were due to the larger inner diameter 


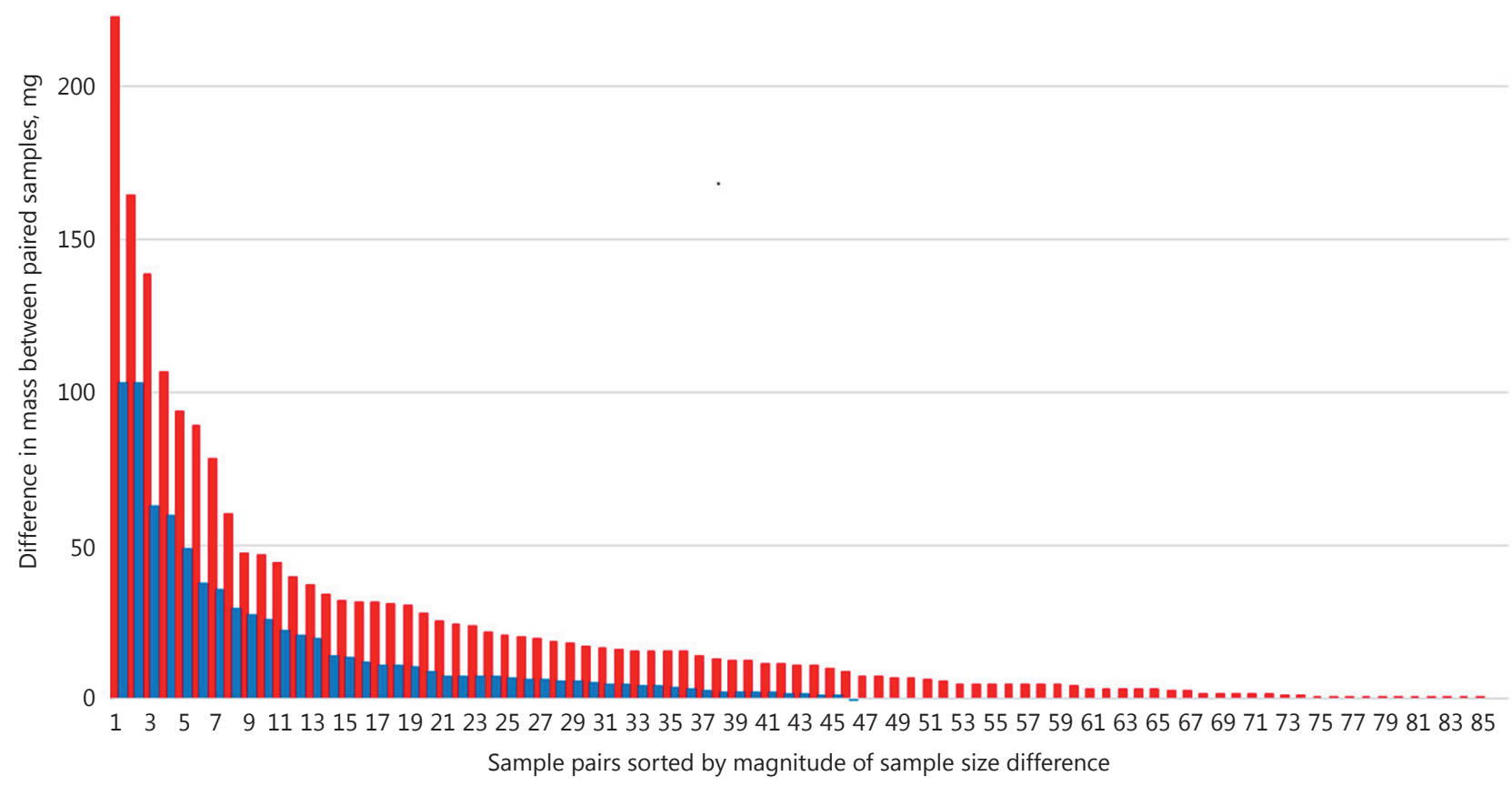

Fig. 3. The difference in sample mass collected by the 19 - and the $22-\mathrm{G}$ needles. There were 85 pairs where the $19-\mathrm{G}$ needle collected a larger sample (red bars) and 46 pairs where the 22-G needle collected a larger sample (blue bars). There was 1 pair where the mass of both samples collected was the same (not included).

Table 3. Tissue types collected by the ViziShot 22-G and the ViziShot FLEX 19-G needles

\begin{tabular}{lccc}
\hline Histology tissue types present in the samples & 19-G needle, $\%$ & 22-G needle, $\%$ & $\begin{array}{l}\text { Wilcoxon } \\
\text { signed-rank } \\
\text { value }\end{array}$ \\
\hline Lymphocytes & & & 0.6422 \\
Sinus reticulum & $12.51 \pm 11.90$ & $13.97 \pm 17.10$ & 0.5509 \\
LN fibrovascular stroma & $0.97 \pm 0.64$ & $0.89 \pm 0.57$ & 0.4773 \\
Infusion granuloma & $14.30 \pm 12.85$ & $12.24 \pm 10.40$ & 0.2351 \\
Crystals & $10.86 \pm 17.96$ & $7.62 \pm 15.87$ & 0.1858 \\
Histiocytic sinus infiltration & $3.92 \pm 6.89$ & $3.00 \pm 6.60$ & 0.8192 \\
Cartilage & $2.95 \pm 3.46$ & $3.32 \pm 4.96$ & 0.0024 \\
Blood (leukocytes) & $4.81 \pm 16.49$ & $0.49 \pm 1.46$ & 0.0573 \\
Adipose tissue & $47.46 \pm 28.01$ & $55.95 \pm 27.84$ & 0.5321 \\
\hline
\end{tabular}

The percentages represent each tissue type recovered from the sampled lymph nodes (LNs) as a proportion of the total tissue recovered. Figures are averages with \pm SD. 
of the needle. It is unlikely that the cartilage contamination was a significant contributor to the larger sample mass collected by the 19-G needle, given that the mass of the $19-G$ needle samples was approximately 34\% greater than that of the samples collected by the $22-\mathrm{G}$ needle, with cartilage comprising only $\sim 4.8 \%$ of the $19-\mathrm{G}$ needle samples.

The ability of the 19-G needle to perform well as a diagnostic tool, while penetrating relatively hard tissue, could make it useful in sampling fibrotic or partially calcified LNs affected by processes like sarcoidosis, some infectious processes (i.e., histoplasmosis) or hematological malignancies (i.e., Hodgkin lymphoma) where the diagnostic performance of EBUS-TBNA is lower than in solid organ malignancy [3, 16-19]. In sarcoidosis, reported EBUSTBNA sensitivity is approximately 0.84 (95\% CI 0.79 0.88). Diagnostic yield ranges from 54 to $93 \%$ with a pooled diagnostic accuracy of approximately $79 \%$. Sensitivity of 0.80 (95\% CI $0.74-0.95)$ has been reported in pulmonary sarcoidosis [20, 21]. In lymphoma, EBUS-TBNAreported yield has also been lower with a wide range of sensitivities ranging between 31 and 91\% [9]. LN fibrosis and calcifications associated with some lymphomas, some infectious processes (i.e., histoplasmosis), and sarcoidosis may be responsible for a higher false-negative rate of EBUS-TBNA sampling and lower diagnostic yield. In the diagnosis of lymphomas, where not only the presence of malignant cells is necessary but also sufficient tissue sample for cytomorphological assessment and ancillary testing (i.e., fluorescent in situ hybridization, immunophenotyping, and immunochemistry), the larger sample collected by the 19-G needle may provide sufficient diagnostic material with fewer passes, therefore decreasing procedure time and the total amount of sedation used.

In infectious granulomatous inflammation like tuberculous lymphadenitis, a different procedure and patientrelated factors including diluted samples, heterogeneity of sampling between different passes, prior antibiotic treatment, and the paucibacillary nature of the disease in immunocompetent patients may be responsible for falsenegative results of EBUS-TBNA [22]. A larger sample collected by the $19-\mathrm{G}$ needle may improve the diagnostic yield in such settings.

Surprisingly, there was a trend towards less blood contamination in the samples collected by the 19-G needle as compared to the samples collected by the smaller lumen $22-\mathrm{G}$ needle. This is counterintuitive as one might expect higher sample blood contamination with a larger gauge needle. Since the clinicians used the same LN sampling technique for both needles, it is unlikely that the sampling method was responsible. It is possible that the new $19-\mathrm{G}$ needle design optimizing needle flexibility, tissue penetration, and sample acquisition contributed to the reduction of sample blood contamination.

While there was no difference in the diagnostic yield in this model of granulomatous inflammation, the sample collected by the 19-G needle was larger. Therefore, we would like to advocate that clinicians choose the needle to sample with based on suspected pathology (i.e., 19-G needle could potentially be selected to sample partially calcified, fibrotic LNs where tissue fibrosis and calcifications may contribute to a lower diagnostic yield, calling for a larger sample) and the amount of tissue needed for diagnosis and/or molecular testing.

In the era of personalized medicine, the molecular signature of tumors impacts prognosis and dictates management. In lung cancer, molecular testing has become the standard of care as targeted therapy in patients with advanced NSCLC harboring EGFR and ALK mutations has been shown to have superior outcomes as compared to the standard, platinum-based chemotherapy [23-25]. Immune checkpoint inhibitors have also shown promising activity with manageable toxicity in patients with NSCLC and may have an important role in the future treatment spectrum with broad patient applicability [26]. EBUS samples have one of the lowest insufficiency rates (4\%) for EGFR and KRAS mutational analysis (compared with CT-FNA - 7.5\%; ultrasound-guided/superficial FNA - 10\%) and can provide sufficient tissue quantity for multigene testing (i.e., p53 mutation, BRAF, and PIK3CA) [7, 27]. However, as new potential targets for treatment of lung and other cancers become identified, tissue acquisition to guide therapy may present a challenge and larger samples may be necessary to meet test standards. Larger, less hemodiluted samples collected by the 19-G needle may offer an advantage over the standard, smaller 22-G needle samples, allowing for multigene testing panels to be performed and perhaps also increase yield in diagnostic assessment of lymphoma and granulomatous inflammation, given a trend towards more frequent identification of granuloma by the 19-G needle in our study.

The debris pellet and smears obtained by both needles did not correlate well with the final LN pathology. This is not surprising, given that the debris pellet sample was a cytological sample of residual material after the solid component of the specimen had been removed. Nonetheless, cellular material was still retrieved by both needles, indicating that even debris left behind, once the macroscopic sample has been separated, may contain viable material that can be processed. It will be important to deter- 
mine whether similar results can be obtained in human subjects with solid organ malignancies and whether needle size makes a difference with regards to cytological analysis.

The study had a few limitations. It was not possible to blind the clinicians to the type of needle used, since given the different needle sizes and associated needle flexibility, stylet thicknesses, and needle locking mechanisms, the clinicians were immediately aware of the needle being used. We attempted to minimize the lack of blinding bias by standardizing the sampling protocol. The generation of a talc granuloma model in swine included measurement of the affected LNs before and after talc injection to assess whether adenopathy had resulted and to confirm that a significant LN size increase had been achieved. However, we did not compare the size of the talc-injected LNs with the size of LNs that had not been injected to ensure that lymphadenopathy was indeed iatrogenic and not part of another pathological process. All injected LNs had an ultrasonographic appearance typical of talc granuloma, from which talc particles were retrieved, alongside diagnostic material. In addition, it would be unlikely for the talc granuloma induction to have failed in all animals with a simultaneous development of mediastinal lymphadenopathy due to an unrecognized pathologic process and in animals that had not exhibited clinical features of any disease throughout the duration of the study. We, therefore, believe that the lack of comparison of the injected and sampled LNs with those that had not been injected and sampled does not invalidate our study. Finally, we assumed that the swine model of granulomatous lymphadenitis can be extrapolated in terms of pathological outcome and EBUS-TBNA performance to that of human granulomatous lymphadenitis. Our study highlights some needle-specific performance features (i.e., the ability to penetrate hard tissue such as cartilage while still being able to collect a good quality diagnostic sample; more blood contamination for the $22-\mathrm{G}$ needle as compared to the 19-G needle). We believe that this information can be useful in designing future human studies. In addition, our study offers guidance to clinicians planning diagnostic EBUS-TBNA as to needle choice depending on suspected pathology.

In conclusion, in this swine model of granulomatous lymphadenopathy, with sampling done by experienced clinicians, the 19-G ViziShot FLEX EBUS-TBNA needle performs similarly in terms of diagnostic yield to the $22-\mathrm{G}$ ViziShot EBUS-TBNA needle, but the sample collected by the 19-G needle is larger and with less blood contamination than the sample collected by the $22-\mathrm{G}$ needle. $\mathrm{Hu}-$ man studies are needed to confirm diagnostic performance in granulomatous inflammation as well as in other types of lymphadenopathy including hematologic and solid organ malignancies.

\section{References}

1 Lindeman NI, Cagle PT, Beasley MB, et al: Molecular testing guideline for selection of lung cancer patients for EGFR and ALK tyrosine kinase inhibitors: guideline from the College of American Pathologists, International Association for the Study of Lung Cancer, and Association for Molecular Pathology. Arch Pathol Lab Med 2013;137:828-860.

2 Czarnecka-Kujawa K, Yasufuku K: Molecular alterations in non-small-cell lung cancer: perspective for targeted therapy and specimen management for the bronchoscopist. Respirology 2014;19:1117-1125.

3 Silvestri GA, Gonzalez AV, Jantz MA, et al: Methods for staging non-small cell lung cancer: diagnosis and management of lung cancer, 3rd ed: American College of Chest Physicians evidence-based clinical practice guidelines. Chest 2013;143:e211S-e250S.

4 Ost DE, Niu J, Elting LS, et al: Quality gaps and comparative effectiveness in lung cancer staging and diagnosis. Chest 2014;145:331345.

19-G versus 22-G EBUS-TBNA Needles:

Diagnostic Comparison
5 Almeida FA, Casal RF, Jimenez CA, et al: Quality gaps and comparative effectiveness in lung cancer staging: the impact of test sequencing on outcomes. Chest 2013;144: 1776-1782.

6 Nakajima T, Yasufuku K, Takahashi R, et al: Comparison of 21-gauge and 22-gauge aspiration needle during endobronchial ultrasound-guided transbronchial needle aspiration. Respirology 2011;16:90-94.

7 Billah S, Stewart J, Staerkel G, et al: EGFR and KRAS mutations in lung carcinoma: molecular testing by using cytology specimens. Cancer Cytopathol 2011;119:111-117.

8 Santis G, Angell R, Nickless G, et al: Screening for EGFR and KRAS mutations in endobronchial ultrasound derived transbronchial needle aspirates in non-small cell lung cancer using COLD-PCR. PLoS One 2011;6:e25191.

9 Kheir F, Itani A, Assasa O, Alraiyes AH: The utility of endobronchial ultrasound-transbronchial needle aspiration in lymphoma. Endosc Ultrasound 2016;5:43-48.
$10 \mathrm{Hu}$ LX, Chen RX, Huang H, et al: Endobronchial ultrasound-guided transbronchial needle aspiration versus standard bronchoscopic modalities for diagnosis of sarcoidosis: a meta-analysis. Chin Med J (Engl) 2016;129: 1607-1615.

11 Chee A, Khalil M, Stather DR, et al: Cytologic assessment of endobronchial ultrasoundguided transbronchial needle aspirates in sarcoidosis. J Bronchology Interv Pulmonol 2012;19:24-28.

12 Tremblay A, Stather DR, Maceachern P, et al: A randomized controlled trial of standard vs endobronchial ultrasonography-guided transbronchial needle aspiration in patients with suspected sarcoidosis. Chest 2009;136:340346.

13 Bang JY, Hawes R, Varadarajulu S: A metaanalysis comparing ProCore and standard fine-needle aspiration needles for endoscopic ultrasound-guided tissue acquisition. Endoscopy 2016;48:339-349. 
14 Tyan C, Patel P, Czarnecka K, et al: Flexible 19-gauge endobronchial ultrasound-guided transbronchial needle aspiration needle: first experience. Respiration 2017;94:52-57.

15 Shackelford C, Long G, Wolf J, et al: Qualitative and quantitative analysis of nonneoplastic lesions in toxicology studies. Toxicol Pathol 2002;30:93-96.

$16 \mathrm{Gu}$ P, Zhao YZ, Jiang LY, et al: Endobronchial ultrasound-guided transbronchial needle aspiration for staging of lung cancer: a systematic review and meta-analysis. Eur J Cancer 2009;45:1389-1396.

17 Adams K, Shah PL, Edmonds L, Lim E: Test performance of endobronchial ultrasound and transbronchial needle aspiration biopsy for mediastinal staging in patients with lung cancer: systematic review and meta-analysis. Thorax 2009;64:757-762.

18 Dong X, Qiu X, Liu Q, Jia J: Endobronchial ultrasound-guided transbronchial needle aspiration in the mediastinal staging of nonsmall cell lung cancer: a meta-analysis. Ann Thorac Surg 2013;96:1502-1507.
19 Sehgal IS, Dhooria S, Aggarwal AN, et al: Endosonography versus mediastinoscopy in mediastinal staging of lung cancer: systematic review and meta-analysis. Ann Thorac Surg 2016;102:1747-1755.

20 Agarwal R, Srinivasan A, Aggarwal AN, Gupta D: Efficacy and safety of convex probe EBUS-TBNA in sarcoidosis: a systematic review and meta-analysis. Respir Med 2012; 106:883-892.

21 Trisolini R, Lazzari Agli L, Tinelli C, et al: Endobronchial ultrasound-guided transbronchial needle aspiration for diagnosis of sarcoidosis in clinically unselected study populations. Respirology 2015;20:226-234.

22 Ko HM, da Cunha Santos G, Darling G, et al: Diagnosis and subclassification of lymphomas and non-neoplastic lesions involving mediastinal lymph nodes using endobronchial ultrasound-guided transbronchial needle aspiration. Diagn Cytopathol 2013;41:10231030.

23 Kwak EL, Bang YJ, Camidge DR, et al: Anaplastic lymphoma kinase inhibition in nonsmall-cell lung cancer. N Engl J Med 2010; 363:1693-1703.
24 Zhou C, Wu YL, Chen G, et al: Erlotinib versus chemotherapy as first-line treatment for patients with advanced EGFR mutation-positive non-small-cell lung cancer (OPTIMAL, CTONG-0802): a multicentre, open-label, randomised, phase 3 study. Lancet Oncol 2011;12:735-742.

25 Lindeman NI, Cagle PT, Beasley MB, et al: Molecular testing guideline for selection of lung cancer patients for EGFR and ALK tyrosine kinase inhibitors: guideline from the College of American Pathologists, International Association for the Study of Lung Cancer, and Association for Molecular Pathology. J Thorac Oncol 2013;8:823-859.

26 Langer CJ: Emerging immunotherapies in the treatment of non-small cell lung cancer (NSCLC): the role of immune checkpoint inhibitors. Am J Clin Oncol 2015;38:422-430.

27 Nakajima T, Yasufuku K, Nakagawara A, et al: Multigene mutation analysis of metastatic lymph nodes in non-small cell lung cancer diagnosed by endobronchial ultrasound-guided transbronchial needle aspiration. Chest 2011;140:1319-1324. 